\title{
HIPK2 sustains inflammatory cytokine production by promoting endoplasmic reticulum stress in macrophages
}

\author{
LONG XU* , HE FANG* ${ }^{*}$ DAYUAN XU and GUANGYI WANG \\ Center of Burns and Trauma, Changhai Hospital, Naval Medical University, Shanghai 200433, P.R. China
}

Received June 7, 2020; Accepted August 21, 2020

DOI: 10.3892/etm.2020.9301

\begin{abstract}
Uncontrolled inflammatory cytokine production by macrophages contributes to numerous conditions, including infection, endotoxemia and sepsis. A previous study proposed that endoplasmic reticulum (ER) stress acts as an essential process in inflammatory cytokine production by macrophages. The present study used a mouse sepsis model and in vitro macrophages to demonstrate that homeodomain-interacting protein kinase 2 (HIPK2) sustained cytokine production in an ER stress-dependent manner. HIPK2 expression was upregulated in the early phase of lipopolysaccharide stimulation. HIPK2 knockdown attenuated IL- 6 and TNF- $\alpha$ production, and p65 phosphorylation in macrophages. Furthermore, the attenuated cytokine production was abolished by the ER stress agonist tunicamycin. The activation of ER stress increased the levels of IL- 6 and TNF- $\alpha$, and the phosphorylation of p65, in macrophages following knockdown of HIPK2. Furthermore, HIPK2 inhibition attenuated the production of IL- 6 and TNF- $\alpha$ in vitro and in vivo. Therefore, HIPK2 sustained inflammatory cytokine production by promoting ER stress in macrophages. Targeting HIPK2 may be a potential strategy for the management of uncontrolled inflammation in clinical settings.
\end{abstract}

\section{Introduction}

Inflammatory diseases, including infection, endotoxemia and sepsis, are a significant burden on perioperative management of patients (1). Inflammation-induced organ dysfunction and failure can result in severe multiple organ dysfunction syndromes (2). The central pathophysiology of inflammation-induced organ dysfunction is the production of

Correspondence to: Professor Guangyi Wang, Center of Burns and Trauma, Changhai Hospital, Naval Medical University, 168 Changhai Road, Shanghai 200433, P.R. China

E-mail: wangguangyi_sh@yeah.net

*Contributed equally

Key words: homeodomain-interacting protein kinase 2, macrophage, inflammation, IL-6, TNF- $\alpha$ an inflammatory cytokine storm caused by uncontrolled and unregulated innate immune responses $(3,4)$. Macrophages produce the majority of cytokines, including IL-6, TNF- $\alpha$ and IL-1 $\beta$, during inflammatory diseases $(3,4)$. Suppressing cytokine production by macrophages can attenuate disease progression and improve outcomes (5). However, the regulation and associated mechanism of cytokine production in macrophages remain largely unknown. The discovery of specific molecules or mechanisms that support macrophage production may reveal potential targets for the management of inflammation.

Previous studies have demonstrated that endoplasmic reticulum (ER) stress serves an essential role in inflammation and cytokine production by macrophages $(6,7)$. The activation of macrophages by lipopolysaccharides (LPS) leads to the unfolded protein response, which results in the activation of three ER stress markers on the ER membrane: Inositol-requiring enzyme $1 \alpha$ (IRE1 $\alpha$ ), PKR-like endoplasmic reticulum kinase (PERK) and activating transcription factor 6 (ATF6) (6). These three molecules regulate subsequent signaling to $\mathrm{NF}-\kappa \mathrm{B}, \mathrm{Bcl}-2$ and nuclear factor erythroid 2 (Nrf2), regulating inflammation, oxidative stress and apoptosis $(8,9)$. Therefore, the activation of ER stress is essential for the inflammatory response of macrophages.

Homeodomain-interacting protein kinase 2 (HIPK2) is a serine/threonine kinase located in the nucleus (10). A previous study has reported that HIPK2 functions as a tumor suppressor and that it promotes apoptosis in response to chemotherapeutic drugs and radiation (10). Furthermore, another previous study proposed that HIPK2 sustains genomic stability by enhancing DNA damage repair signaling (11). Apart from the role of HIPK2 that has been elucidated within oncological research, a recent study demonstrated that HIPK2 deficiency leads to the impaired production of type I interferon in macrophages during antiviral immunity (12). However, the function and mechanism of HIPK2 in anti-microbial immunity remain poorly investigated.

The present study investigated the function of HIPK2 in LPS-stimulated macrophages and the underlying mechanism of cytokine production. Furthermore, the expression levels of HIPK2, effects of HIPK2 knockdown and the crucial mechanism of its regulation of cytokines in LPS-stimulated macrophages were investigated. 


\section{Materials and methods}

Animals and models. A total of $60 \mathrm{C} 57 \mathrm{BL} / 6$ male mice (weight, 20-25 g; age, 6-8 weeks) were purchased from Shanghai SLAC Laboratory Animal Co., Ltd. All mice were housed at a temperature of $18-22^{\circ} \mathrm{C}$ with a relative humidity of 50-60\% and 12-h light-dark cycles, with free access to food and water. The Ethics Committee of Changhai Hospital (Shanghai, China) approved the animal experiments. For the murine LPS-challenge model, each mouse was intraperitoneally injected with 4,5,6,7-tetrabromo-2-(1H-imidazol-2-yl) isoindoline-1,3-dione (tBID) (10 ng/g; cat. no. HY-100464; MedChemExpress) diluted in $400 \mu 1$ PBS. For tBID and tunicamycin (TM; cat. no. HY-A0098; MedChemExpress) co-treatment, tBID $(10 \mathrm{ng} / \mathrm{g})$ and TM $(0.5 \mathrm{mg} / \mathrm{kg})$ were injected intraperitoneally $30 \mathrm{~min}$ prior to LPS challenge. Following this, mice were intraperitoneally injected with $10 \mathrm{mg} / \mathrm{kg}$ LPS (Escherichia coli O111: B4; Sigma-Aldrich; Merck KGaA) diluted in $300 \mu \mathrm{l} \mathrm{PBS}$. At $6 \mathrm{~h}$ after the LPS injection, mice were anesthetized with $4 \%$ sevoflurane (Abbott Pharmaceutical Co. Ltd.) and serum (stored in $-80^{\circ} \mathrm{C}$ ) was harvested through the postocular venous plexus. Cervical dislocation was used for euthanasia.

Preparation of bone marrow-derived macrophages (BMDMs) and peritoneal macrophages (PMs). For BMDM generation, mouse femoral tissues were isolated and the bone marrow was flushed with $3 \mathrm{ml}$ normal saline (NS). Following red blood cell lysis, bone marrow cells were resuspended at $2.4 \times 10^{6}$ cells $/ \mathrm{ml}$ in DMEM (Hyclone; GE Healthcare Life Science) supplemented with $10 \%$ FBS (Gibco; Thermo Fisher Scientific, Inc.) and $30 \mathrm{ng} / \mathrm{ml}$ granulocyte-macrophage colony-stimulating factor (PeproTech, Inc.). The culture medium was changed every 2 days, and after $5-6$ days of culturing $\left(37^{\circ} \mathrm{C}\right.$ and $5 \%$ $\mathrm{CO}_{2}$ ), cells were subjected to further experiments.

For PM generation, each mouse was injected intraperitoneally with thioglycollate (BD Biosciences). At day 3 post-injection, peritoneal lavage was performed with $5 \mathrm{ml}$ NS. Cells were resuspended at $2-4 \times 10^{6}$ cells $/ \mathrm{ml}$ and cultured in RPMI 1640 (Hyclone; GE Healthcare Life Science) culture medium supplemented with 10\% FBS. LPS stimulation was achieved by adding LPS $(100 \mathrm{ng} / \mathrm{ml})$ at the indicated time points in each experiment, and the supernatant or mRNA and protein were harvested for further analysis. TM $(10 \mu \mathrm{g} / \mathrm{ml}$ in vitro or $0.5 \mathrm{mg} / \mathrm{kg}$ in vivo) was added $30 \mathrm{~min}$ prior to LPS stimulation in vitro and in vivo.

Reverse transcription-quantitative PCR analysis. For mRNA analysis, cells were stimulated with LPS $(100 \mathrm{ng} / \mathrm{ml})$ for 0.5 , $1,3,6 \mathrm{~h}$ and then the cells were washed with PBS and the total RNA were extracted. Total RNA from macrophages (BMDMs for expression and inhibitor experiments and PMs for siRNA interference experiments) was extracted using TRIzol $^{\circledR}$ reagent (cat. no. 10296010; Thermo Fisher Scientific, Inc.). Complementary DNA templates were obtained by reverse transcription $\left(50^{\circ} \mathrm{C}\right.$ for $45 \mathrm{~min}$ and at $85^{\circ} \mathrm{C}$ for $\left.5 \mathrm{~min}\right)$ in a $10 \mu \mathrm{l}$ reaction volume containing $1 \mu \mathrm{g}$ total RNA, oligo (dT) primers and a reverse transcription premix (Takara, Bio, Inc.). Quantitative PCR was performed using a SYBR-Green PCR system and an ABI 7500 Thermal Cycler (High-Capacity
cDNA Reverse Transcription kit, cat. no. 4374967; PowerUp ${ }^{\mathrm{TM}}$ SYBR $^{\mathrm{TM}}$ Green Master Mix, cat. no. A25742; Thermo Fisher Scientific, Inc.) according to the manufacturer's protocol. SYBR-Green reagents were purchased from Thermo Fisher Scientific, Inc. The thermocycling conditions were as follows: $95^{\circ} \mathrm{C}$ for $3 \mathrm{~min}$, followed by 40 cycles of denaturation at $95^{\circ} \mathrm{C}$ for $10 \mathrm{sec}$, annealing at $60^{\circ} \mathrm{C}$ for $5 \mathrm{sec}$ and extension at $72^{\circ} \mathrm{C}$ for $10 \mathrm{sec}$. mRNA levels were normalized to the mRNA level of $\beta$-actin, which was used as the internal control. The primers used were as follows: $\beta$-actin, forward, 5'-CTCCATCCTGGC CTCGCTGT-3' and reverse, 5'-GCTGTCACCTTCACCGTT CC-3'; PERK forward, 5'-TCCCCTAGATCCCCTGAACTT-3' and reverse, 5'-TGGAGTGTCTGATCTTCACTGA-3'; IL-6 forward, 5'-GGCGGATCGGATGTTGTGAT-3' and reverse, 5'-GGACCCCAGACAATCGGTTG-3'; TNF- $\alpha$ forward, 5'-GGAACACGTCGTGGGATAATG-3' and reverse, 5'-GGC AGACTTTGGATGCTTCTT-3'; IRE1 $\alpha$ forward, 5'-TTGAGA GAGCTTTTACCAGCAG-3' and reverse, 5'-ACCAGGACC TGACGGATGT-3'; and ATF6, forward, 5'-TGGAGCAGG ATGTCCCGTT-3' and reverse, 5'-CTGTGGAAAGATGTG AGGACTC-3'. Relative mRNA levels were determined using the $2^{-\Delta \Delta C q}$ method (13) and $\beta$-actin was used as the internal control.

ELISA analysis. Blood was centrifuged at $845 \mathrm{x}$ g for $15 \mathrm{~min}$ and serum was collected. Serum levels of IL- 6 and TNF- $\alpha$ were analyzed using Quantikine IL-6 and TNF- $\alpha$ ELISA kits (cat. nos. M6000B and MTA00B; R\&D Systems, Inc.) according to the manufacturer's protocol.

Small interfering RNA (siRNA) interference. Mouse PMs at a confluence of $80 \%$ were cultured in half of the final total culture volume in FBS-free RPMI-1640 or DMEM and transfected with $3 \mathrm{ng} / \mathrm{ml}$ HIPK2 siRNA (Gene Pharma; sense, 5'-GGA GUUCAUUGACCUGUUAAA-3' and anti-sense, 5'-UAA CAGGUCAAUGAACUCCCG-3') or $6 \mathrm{ng}(3 \mathrm{ng} / \mathrm{ml})$ control siRNA (Shanghai GenePharma Co., Ltd.; sense, 5'-UUCUCC GAACGUGUCACGUTT-3' and anti-sense, 5'-ACGUGACAC GUUCGGAGAATT-3') in 6-well plates using INTERFEREin (Invitrogen; Thermo Fisher Scientific, Inc.) for $6 \mathrm{~h}\left(37^{\circ} \mathrm{C}\right)$ according to the manufacturer's protocol. Subsequently, the other half of the complete culture medium was added and the cells were cultured $\left(37^{\circ} \mathrm{C}\right)$ for a total of $48 \mathrm{~h}$. After $48 \mathrm{~h}$, cells were subjected to further stimulation or experiments.

Western blotting. Cells were lysed in RIPA (Beyotime Institute of Biotechnology). The protein concentration was determined using a BCA protein assay kit (Thermo Fisher Scientific, Inc.). A total of $30 \mu \mathrm{g}$ protein/lane was subjected to $10 \%$ SDS-PAGE and transferred to PVDF membranes (Merck $\mathrm{KGaA}$ ). The membranes were blocked with $5 \%$ non-fat milk in PBS with $0.05 \%$ Tween-20 (pH 7.5) at room temperature for $30 \mathrm{~min}$. Membranes were then immunoblotted with the primary antibodies $(1: 2,000)$ for $4 \mathrm{~h}$ at room temperature or at $4^{\circ} \mathrm{C}$ overnight. The primary antibodies used in the present study were: Anti-mouse HIPK2 (cat. no. 5091; Cell Signaling Technology, Inc.), phosphorylated (p-) p65 (cat. no. 3033; Cell Signaling Technology, Inc.), p65 cat. no. 9460; Cell Signaling Technology, Inc.), phosphorylated (p-)PERK (cat. no. 3179S; Cell Signaling Technology, Inc.), PERK (cat. no. 3192S; 

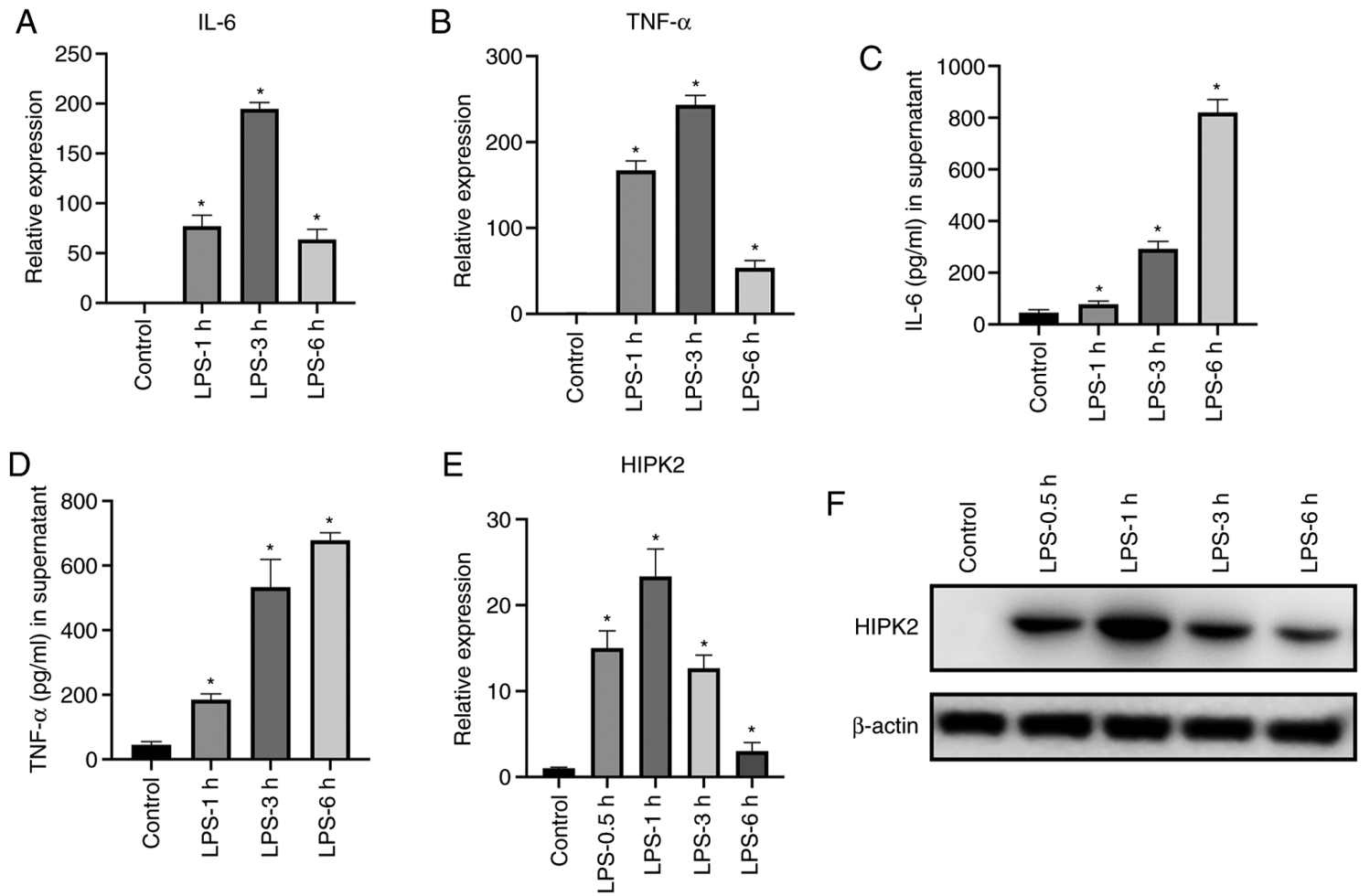

Figure 1. HIPK2 expression is upregulated following LPS stimulation in macrophages. RT-qPCR analysis of (A) IL-6 and (B) TNF- $\alpha$, ELISA analysis of (C) IL-6 and (D) TNF- $\alpha$ in supernatants, and (E) RT-qPCR and (F) western blot analysis of HIPK2 in LPS-stimulated bone marrow-derived macrophages at the indicated time points ( $\mathrm{n}=3$ per group). " $\mathrm{P}<0.05$ vs. control. HIPK2, homeodomain-interacting protein kinase 2; LPS, lipopolysaccharides; RT-qPCR, reverse transcription-quantitative PCR.

Cell Signaling Technology, Inc.), p-IRE1 $\alpha$ (cat. no. ab48187; Abcam), IRE1 $\alpha$ (cat no. 3294S; Cell Signaling Technology, Inc.), ATF6 (cat. no. ab122897; Abcam) and $\beta$-actin (cat. no. ab8227; Abcam). Subsequently, membranes were incubated with horseradish peroxidase-conjugated secondary antibodies (1:3,000) (cat. nos. 7074 and 7076; Cell Signaling Technology, Inc.). Finally, the blots were visualized using SuperSignal ${ }^{\mathrm{TM}}$ West Atto Ultimate Sensitivity Substrate (Thermo Fisher Scientific, Inc.).

Statistical analysis. Data from three independent experiments were repeated and are presented as the mean \pm standard deviation. Student's t-test was used to analyze differences between two groups. One-way ANOVA was used to analyze differences among multiple groups. Sidak's multiple comparisons test or Dunnett's post hoc test were used as the post hoc tests. Analysis was performed with Prism software (version no. 8; GraphPad Software, Inc.). $\mathrm{P}<0.05$ was considered to indicate a statistically significant difference.

\section{Results}

HIPK2 expression is upregulated by LPS stimulation in macrophages. To investigate the expression levels of HIPK2 during inflammatory cytokine production in macrophages, the mRNA and protein expression levels of cytokines and HIPK2 were analyzed. The results demonstrated that following LPS stimulation, IL-6 and TNF- $\alpha$ were significantly upregulated at the mRNA (Fig. 1A and B) and protein (Fig. 1C and D) levels. The peak transcription of IL- 6 and TNF- $\alpha$ was observed at $3 \mathrm{~h}$ following stimulation. Furthermore, the expression levels of HIPK2 were elevated following LPS stimulation (Fig. $1 \mathrm{E}$ and F), and the peak production was observed at 0.5 and $1 \mathrm{~h}$ after LPS stimulation at the mRNA and protein levels, respectively. These results demonstrated that HIPK2 expression is upregulated earlier than the expression of cytokines, indicating a potential role of HIPK2 in the regulation of cytokine production in macrophages.

HIPK2 knockdown suppresses IL- 6 and TNF- $\alpha$ production in macrophages. The potential role of HIPK 2 in the cytokine production by macrophages was investigated. siRNA was used to knock down HIPK2 expression, and the effects on macrophages were investigated. The results revealed that HIPK 2 was successfully knocked down at the mRNA level in LPS stimulation (Fig. 2A) and protein (Fig. 2B) levels. Furthermore, the results demonstrated that the phosphorylation of $\mathrm{p} 65$, the effective component of NF- $\kappa \mathrm{B}$ (3), was markedly downregulated following HIPK2 knockdown (Fig. 2B) in the presence of LPS. Similarly, IL-6 (Fig. 2C) and TNF- $\alpha$ (Fig. 2D) production was suppressed following HIPK2 knockdown. Therefore, these results indicated that HIPK2 may serve an essential role in cytokine production during LPS stimulation in macrophages.

HIPK2 knockdown suppresses ER stress in LPS-stimulated macrophages. The mechanism by which HIPK 2 promoted cytokine production was investigated. A recent study has demonstrated the crucial role of ER stress in macrophage inflammation (6). Inhibition of ER stress alleviates inflammation and cytokine production by macrophages (7). The results 


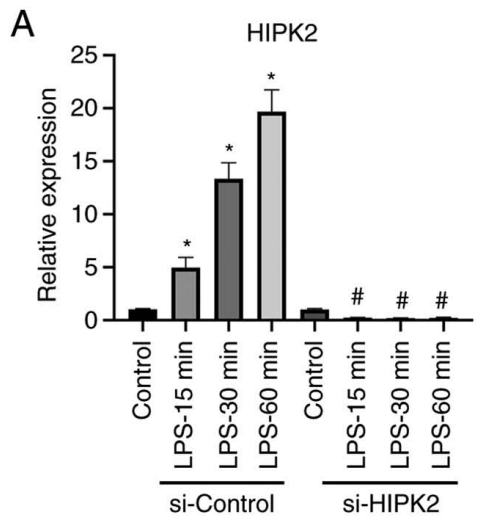

$\mathrm{C}$

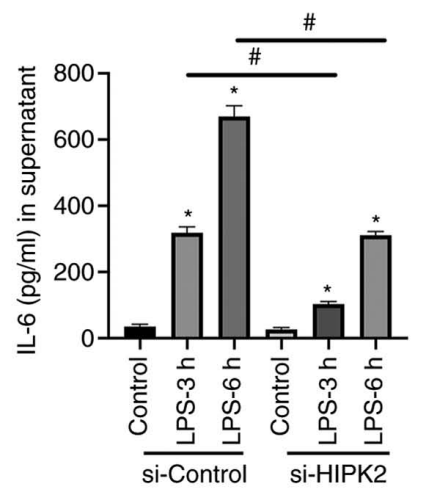

B

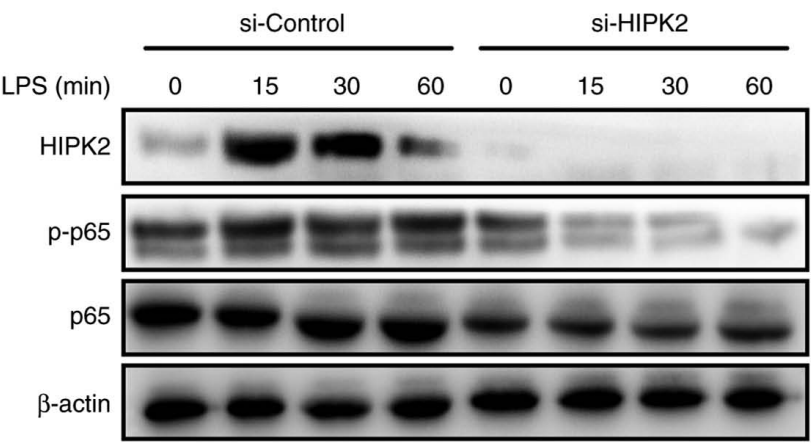

$\mathrm{D}$

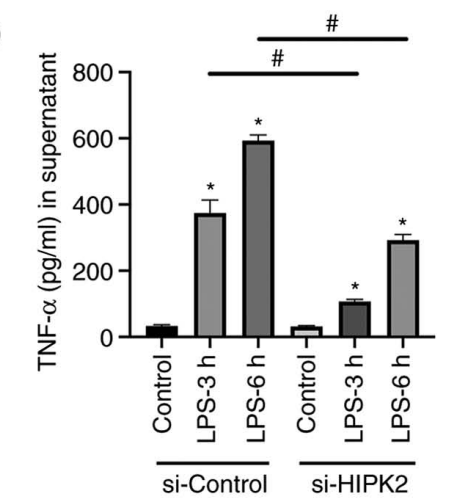

Figure 2. HIPK2 knockdown suppresses IL-6 and TNF- $\alpha$ production in macrophages. (A) Knockdown efficacy of si-HIPK2 and controls at the mRNA level in LPS-stimulated PMs at the indicated time points $(n=3)$. (B) Western blot analysis of HIPK2 following siRNA interference, and levels of p-p65 and p65 in PMs (three independent repeated experiments were performed). ELISA analysis of (C) IL-6 and (D) TNF- $\alpha$ in the supernatant following HIPK2 knockdown in LPS-stimulated PMs $(n=4) .{ }^{*} \mathrm{P}<0.05$ vs. control; ${ }^{\#} \mathrm{P}<0.05$ vs. corresponding si-controls in the same group. HIPK2, homeodomain-interacting protein kinase 2; si, small interfering RNA; PMs, peritoneal macrophages; p-, phosphorylated; LPS, lipopolysaccharides.

demonstrated that, after the interference of HIPK2, the mRNA level of IRE1 $\alpha$ in 1 and $3 \mathrm{~h}$ after LPS stimulation, PERK and ATF6 in $3 \mathrm{~h}$ after LPS stimulation were downregulated (Fig. 3A-C). The results also revealed that the protein level of p-IRE1 $\alpha, p$-PERK and ATF6 at 15, 30 and 60 min after the LPS stimulation were downregulated (Fig. 3D), indicating that ER stress may be a potent mechanism of HIPK2 for the promotion of cytokine production by macrophages.

Activation of ER stress restores IL-6 and TNF- $\alpha$ production following HIPK2 knockdown in LPS-stimulated macrophages. To investigate the essential role of ER stress in HIPK2-mediated cytokine production, TM, an ER stress agonist (14), was used to establish the effects of HIPK2 knockdown in macrophages. The results revealed that while HIPK 2 knockdown attenuated IL- 6 and TNF- $\alpha$ production, the application of TM, though slightly decreased IL- 6 and TNF- $\alpha$ compared with the control, abolished the attenuation of cytokine production (Fig. 4A and B) and restored the phosphorylation level of p65 (Fig. 4C). Therefore, these results indicated that ER stress is an essential process for HIPK2-mediated cytokine production by macrophages.

HIPK2 inhibition suppresses IL- 6 and TNF- $\alpha$ production in vitro and in vivo. To investigate whether HIPK 2 was a potent anti-inflammatory agent. The effects of the HIPK2 inhibitor, tBID, in LPS-stimulated macrophages and LPS-challenged mice were investigated. The results demonstrated that, in cultured macrophages, tBID treatment suppressed IL-6 (Fig. 5A) and TNF- $\alpha$ (Fig. 5B) levels in the LPS treatment groups. Furthermore, in LPS-challenged mice, inhibition of HIPK2 was associated with favorable outcomes in terms of attenuating the serum levels of IL-6 (Fig. 5C) and TNF- $\alpha$ (Fig. 5D). TM was used to activate ER stress in the presence of tBID to investigate whether the ER agonist restored cytokine production following HIPK2 inhibition. The results demonstrated that IL-6 (Fig. 5E) and TNF- $\alpha$ (Fig. 5F) levels were elevated compared with the LPS group without TM treatment, and the effects of HIPK2 inhibition were decreased by TM treatment. The TM treatment was also demonstrated to elevate IL-6 and TNF- $\alpha$ levels (Fig. 5E and F). Collectively, these results indicated that HIPK2 inhibition suppressed cytokine production in vitro and in vivo, suggesting its potential role in anti-inflammation management.

\section{Discussion}

Uncontrolled activation of inflammation and subsequent cytokine storm are essential contributors to inflammatory organ dysfunction and failure (15). Endotoxemia and sepsis are usually initiated from the overactivation of macrophages and the following excessive production of inflammatory cytokines $(4,16)$. Therefore, an in-depth understanding of cytokine production regulation may provide an important mechanism for the management of inflammation. The present study revealed that HIPK2 served a positive role in 
A

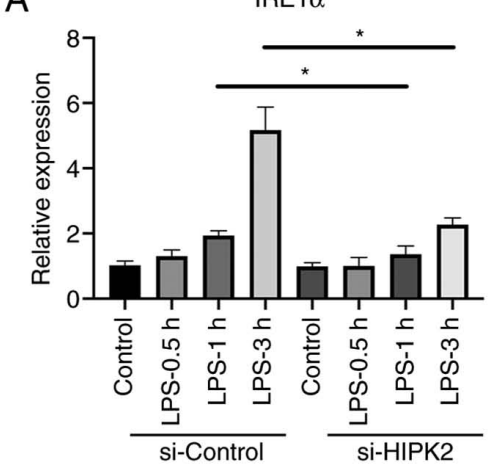

C

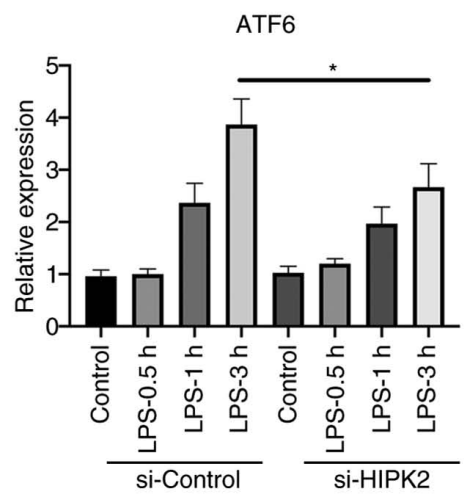

B

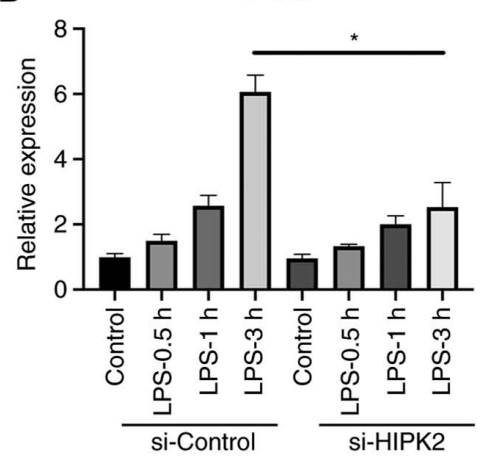

D

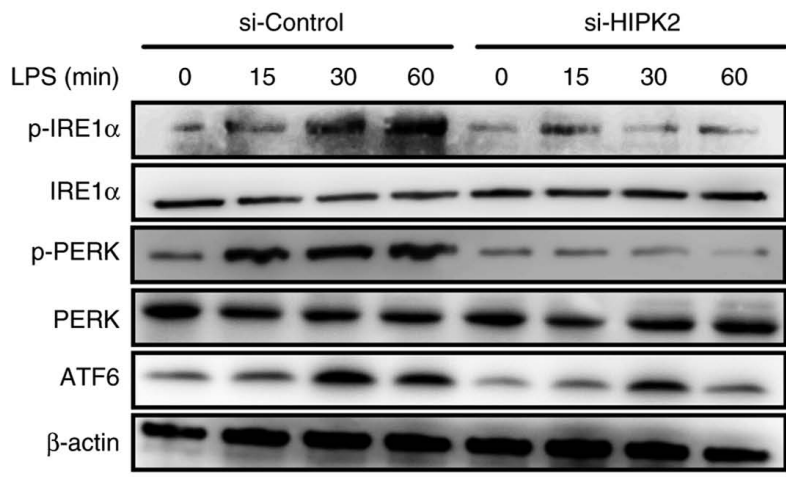

Figure 3. HIPK2 knockdown suppresses endoplasmic reticulum stress in LPS-stimulated macrophages. Reverse transcription-quantitative PCR analysis of (A) IRE1 $\alpha$, (B) PERK and (C) ATF6 expression following HIPK2 interference in LPS-stimulated PMs (n=3). (D) Western blot analysis of p-IRE1 $\alpha$, IRE1 $\alpha$, p-PERK, PERK and ATF6 following HIPK2 interference in LPS-stimulated PMs. " $\mathrm{P}<0.05$ as indicated. HIPK2, homeodomain-interacting protein kinase 2; LPS, lipopolysaccharides; IRE1 $\alpha$, inositol-requiring enzyme 1 $\alpha$; PERK, PKR-like endoplasmic reticulum kinase; ATF6, activating transcription factor 6; PMs, peritoneal macrophages; p-, phosphorylated; si, small interfering RNA.

A

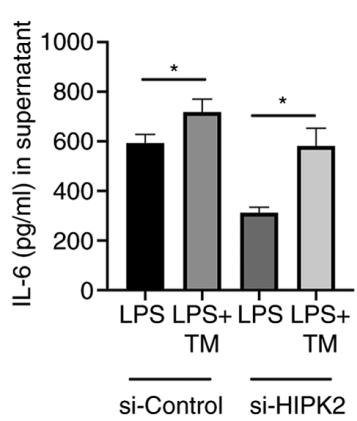

$\mathrm{B}$

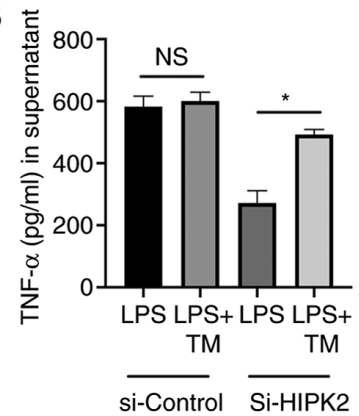

C

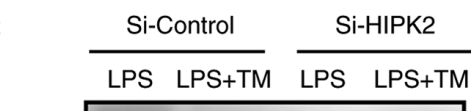

p-p65

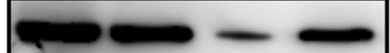

p65

$\beta$-actin

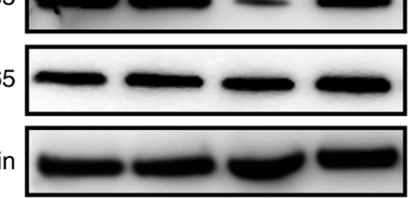

Figure 4. Activation of endoplasmic reticulum stress restores IL-6 and TNF- $\alpha$ production following HIPK2 knockdown in LPS-stimulated macrophages. ELISA analysis of (A) IL-6 and (B) TNF- $\alpha$ levels in the supernatants with or without TM treatment in PMs following HIPK2 knockdown (n=3). (C) Western blot analysis of p-p65 and p65 levels in PMs treated with or without TM following HIPK2 knockdown. "P $<0.05$ as indicated. HIPK2, homeodomain-interacting protein kinase 2; LPS, lipopolysaccharides; PMs, peritoneal macrophages; TM, tunicamycin; p-, phosphorylated; si, small interfering RNA; NS, not significant.

maintaining LPS-induced inflammation of macrophages and that HIPK2 knockdown led to the attenuation of cytokine production. Two types of macrophages were used in the present study. BMDMs and PMs are regarded as models for macrophage investigation $(2,3)$. The present study used BMDMs to analyze the expression levels of HIPK 2 in in vitro inhibitor experiments and in in vitro experiments with HIPK2 interference, while PMs were used to ensure the interference efficacy (4). The results were in accordance with a previous study that reported that HIPK2 was crucial in maintaining type I interferon production in antiviral immunity of macrophages (12). The present study highlighted that HIPK2 may be a potential target to control excessive cytokine production by overactivated macrophages.

The results demonstrated that ER stress served an essential role in HIPK2-mediated cytokine production. Through HIPK2 knockdown and ER stress agitation, the present study revealed that HIPK2-promoted cytokine production was dependent on the activation of ER stress, indicating that HIPK2 may activate ER stress to promote cytokine production. ER stress has been reported to serve a crucial role in macrophagic inflammation (17). Previous studies have demonstrated that the activation of ER stress promotes NF- $\mathrm{B}$ and Nrf2 activation, which further promotes the transcription of pro-inflammatory genes, 

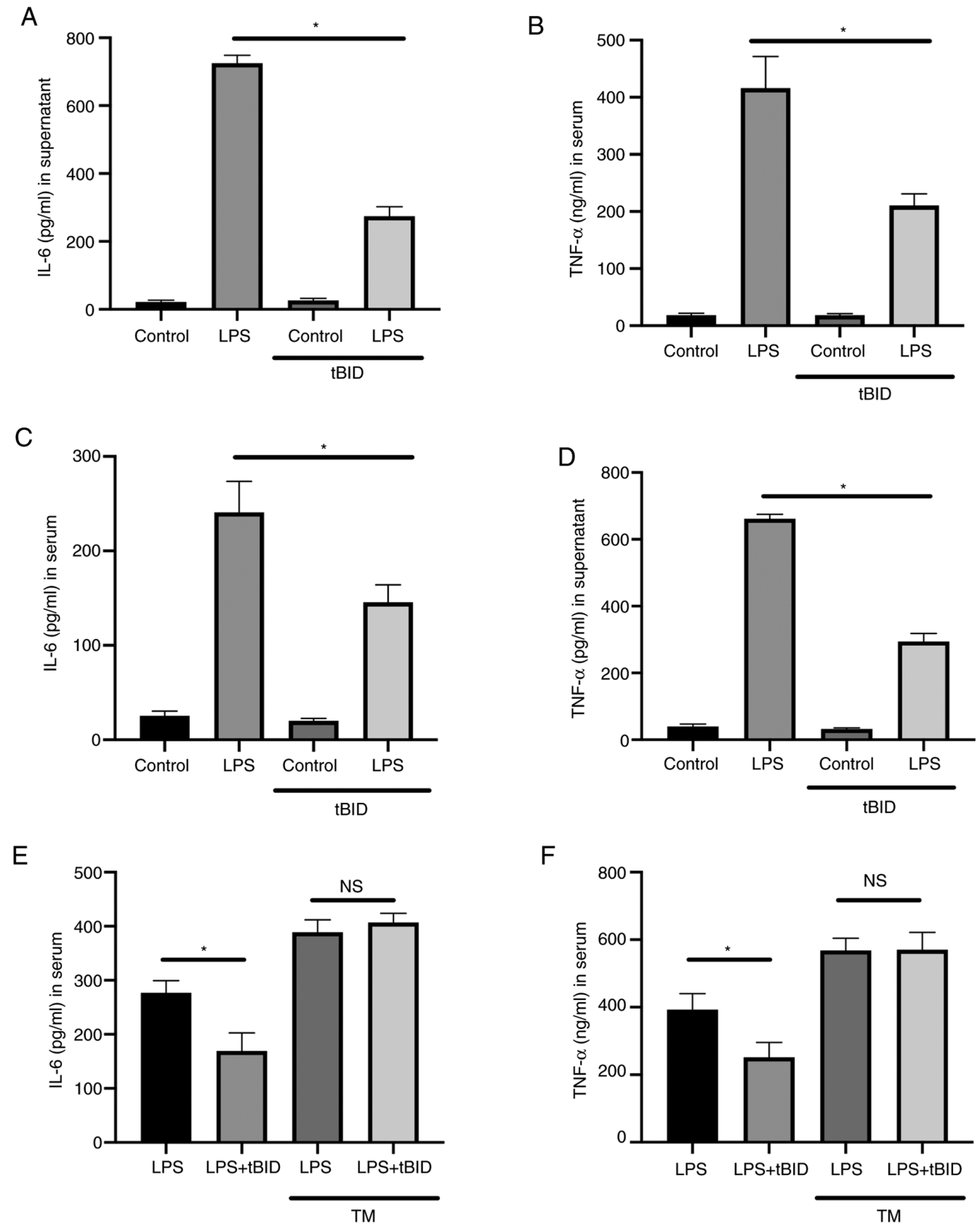

Figure 5. HIPK2 inhibition suppresses IL-6 and TNF- $\alpha$ production in vitro and in vivo. ELISA analysis of (A) IL-6 and (B) TNF- $\alpha$ levels in the serum of mice treated with or without tBID and LPS stimulation for $6 \mathrm{~h}(\mathrm{n}=3)$. ELISA analysis of (C) IL-6 and (D) TNF- $\alpha$ levels in the supernatants of bone marrow-derived macrophages treated with or without tBID and challenged with LPS for $6 \mathrm{~h}(\mathrm{n}=6)$. ELISA analysis of (E) IL- 6 and (F) TNF- $\alpha$ levels in the serum of mice treated with or without tBID and TM (three independent repeated experiments). " $\mathrm{P}<0.05$ as indicated. HIPK2, homeodomain-interacting protein kinase 2; LPS, liposaccharides; TM, tunicamycin; NS, not significant; tBID (4,5,6,7-tetrabromo-2-(1H-imidazol-2-yl)isoindoline-1,3-dione).

such as p65 and Erk, and oxidative stress (6,9). Additionally, ER stress promotes mitochondrial stress and initiates respiratory burst in mitochondria, promoting the production of reactive oxygen species in macrophages (18-20). Numerous molecules, such as ALDH2 and NOD1, regulate ER stress through exogenous receptor-ligand reactions and intrinsic regulation $(20,21)$. The present study indicated a potential role in regulating ER stress. Investigation of the specific mechanism of the regulation could be a topic of interest in future studies. However, for the in vivo experiments, since the present study was not able to select targeted macrophages, the alteration of these markers could not be measured. LPS challenge results in systematic inflammation and macrophages and monocyte-macrophages can infiltrate organs and tissues (22). This is in accordance with a previous study which demonstrated the in vivo expression of certain macrophage genes, including v-ets erythroblastosis virus E26 oncogene homolog 1 and 2, and presented the results as serum cytokine levels following the inhibition or activation of various molecules, including Erk or interleukin 1 receptor-associated kinase $\mathrm{M}$ (3), which the present study investigated using tBID and TM in vivo.

Since cytokine production relies on HIPK2 expression in macrophages, the present study investigated the potential effects of HIPK2 inhibition in an LPS-stimulated model 
in vitro and in vivo. However, although the present study was a preliminary investigation and further studies are required to address the effects in different inflammatory models, the present study indicated the therapeutic effects of HIPK2 inhibition in anti-inflammation management. HIPK2 may be a promising target for the treatment of inflammatory diseases.

In summary, the present study reported the crucial role of HIPK2 in promoting cytokine production in response to LPS in macrophages. Furthermore, the results demonstrated that HIPK2-mediated cytokine production was dependent on the activation of ER stress. Targeting HIPK2 may be a potential strategy for the management of inflammation.

\section{Acknowledgements}

Not applicable.

\section{Funding}

The present study was supported by the National Natural Science Foundation of China (grant nos. 8167080319 and 81701899).

\section{Availability of data and materials}

The datasets used and/or analyzed during the current study are available from the corresponding author on reasonable request.

\section{Authors' contributions}

LX and HF performed the majority of experiments and prepared the manuscript. DX assisted in animal experiments and contributed to the preparation of the manuscript. GW designed the present study and reviewed the manuscript. All authors read and approved the final manuscript.

\section{Ethics approval and consent to participate}

The Ethics Committee of Changhai Hospital approved the animal experiments.

\section{Patient consent for publication}

Not applicable.

\section{Competing interests}

The authors declare that they have no competing interests.

\section{References}

1. Thorburn AN, Macia L and Mackay CR: Diet, metabolites, and 'western-lifestyle' inflammatory diseases. Immunity 40: 833-42, 2014.

2. Lamkanfi M, Sarkar A, Vande Walle L, Vitari AC, Amer AO, Wewers MD, Tracey KJ, Kanneganti TD and Dixit VM: Inflammasome-dependent release of the alarmin HMGB1 in endotoxemia. J Immunol 185: 4385-4392, 2010.

3. Ma X, Jiang Z, Li N, Jiang W, Gao P, Yang M, Yu X, Wang G and Zhang Y: Ets2 suppresses inflammatory cytokines through MAPK/NF- $\kappa \mathrm{B}$ signaling and directly binds to the IL-6 promoter in macrophages. Aging (Albany NY) 11: 10610-10625, 2019.
4. Xu M, Jiang Z, Wang C, Li N, Bo L, Zha Y, Bian J, Zhang Y and Deng X: Acetate attenuates inflammasome activation through GPR43-mediated $\mathrm{Ca}(2+)$-dependent NLRP3 ubiquitination. Exp Mol Med 51: 83, 2019.

5. Wen M, Ma X, Cheng H, Jiang W, Xu X, Zhang Y, Zhang Y, Guo Z, $\mathrm{Yu} \mathrm{Y}, \mathrm{Xu} \mathrm{H}$, et al: Stk38 protein kinase preferentially inhibits TLR9-activated inflammatory responses by promoting MEKK2 ubiquitination in macrophages. Nat Commun 6: 7167, 2015.

6. Zeeshan HM, Lee GH, Kim HR and Chae HJ: Endoplasmic reticulum stress and associated ROS. Int J Mol Sci 17: 327, 2016.

7. Sicari D, Delaunay-Moisan A, Combettes L, Chevet E and Igbaria A: A guide to assessing endoplasmic reticulum homeostasis and stress in mammalian systems. FEBS J 287: 27-42, 2020.

8. Fu S, Yang L, Li P, Hofmann O, Dicker L, Hide W, Lin X, Watkins SM, Ivanov AR and Hotamisligil GS: Aberrant lipid metabolism disrupts calcium homeostasis causing liver endoplasmic reticulum stress in obesity. Nature 473: 528-531, 2011.

9. Lebeaupin C, Vallée D, Hazari Y, Hetz C, Chevet E and Bailly-Maitre B: Endoplasmic reticulum stress signalling and the pathogenesis of non-alcoholic fatty liver disease. J Hepatol 69: 927-947, 2018.

10. Akaike Y, Kuwano Y, Nishida K, Kurokawa K, Kajita K, Kano S, Masuda K and Rokutan K: Homeodomain-interacting protein kinase 2 regulates DNA damage response through interacting with heterochromatin protein 1 $\gamma$. Oncogene 34: 3463-3473, 2015.

11. Torrente L, Sanchez C, Moreno R, Chowdhry S, Cabello P, Isono K, Koseki H, Honda T, Hayes JD, Dinkova-Kostova AT and de la Vega L: Crosstalk between NRF2 and HIPK2 shapes cytoprotective responses. Oncogene 36: 6204-6212, 2017.

12. Cao L, Yang G, Gao S, Jing C, Montgomery RR, Yin Y, Wang P, Fikrig E and You F: HIPK2 is necessary for type I interferon-mediated antiviral immunity. Sci Signal 12: eaau4604, 2019.

13. Livak KJ and Schmittgen TD: Analysis of relative gene expression data using real-time quantitative PCR and the 2(-Delta Delta C(T)) method. Methods 25: 402-408, 2001.

14. Xu Z, Duan F, Lu H, Abdulkadhim Dragh M, Xia Y, Liang H and Hong L: UBIAD1 suppresses the proliferation of bladder carcinoma cells by regulating H-Ras intracellular trafficking via interaction with the C-terminal domain of H-Ras. Cell Death Dis 9: 1170, 2018.

15. Zhang XW, Meng X, Chen YY, Leng SX and Zhang HY: The biology of aging and cancer frailty, inflammation, and immunity. Cancer J 23: 201-205, 2017.

16. Jiang Z, Bo L, Meng Y, Wang $\mathrm{C}$, Chen $\mathrm{T}$, Wang $\mathrm{C}$, Yu X and Deng $\mathrm{X}$ : Overexpression of homeodomain-interacting protein kinase 2 (HIPK2) attenuates sepsis-mediated liver injury by restoring autophagy. Cell Death Dis 9: 847, 2018.

17. Kim O-K, Jun W and Lee J: Mechanism of ER stress and inflammation for hepatic insulin resistance in obesity. Ann Nutr Metab 67: 218-227, 2015.

18. Levada K, Guldiken N, Zhang X, Vella G, Mo FR, James LP, Haybaeck J, Kessler SM, Kiemer AK, Ott T, et al: Hsp72 protects against liver injury via attenuation of hepatocellular death, oxidative stress, and JNK signaling. J Hepatol 68: 996-1005, 2018.

19. Li L, Wang H, Zhang J, Sha Y, Wu F, Wen S, He L, Sheng L, You Q, Shi M, et al: SPHK1 deficiency protects mice from acetaminophen-induced ER stress and mitochondrial permeability transition. Cell Death Differ 27: 1924-1937, 2020.

20. Keestra-Gounder AM, Byndloss MX, Seyffert N, Young BM, Chávez-Arroyo A, Tsai AY, Cevallos SA, Winter MG, Pham OH, Tiffany CR, et al: NOD1 and NOD2 signalling links ER stress with inflammation. Nature 532: 394-397, 2016.

21. Kimura Y, Inoue A, Hangai S, Saijo S, Negishi H, Nishio J, Yamasaki S, Iwakura Y, Yanai $\mathrm{H}$ and Taniguchi $\mathrm{T}$ : The innate immune receptor Dectin-2 mediates the phagocytosis of cancer cells by Kupffer cells for the suppression of liver metastasis. Proc Natl Acad Sci USA 113: 14097-14102, 2016.

22. Pang J, Peng H, Wang S, Xu X, Xu F, Wang Q, Chen Y, Barton LA, Chen Y, Zhang Y and Ren J: Mitochondrial ALDH2 protects against lipopolysaccharide-induced myocardial contractile dysfunction by suppression of ER stress and autophagy. Biochim Biophys Acta Mol Basis Dis 1865: 1627-1641, 2019.

This work is licensed under a Creative Commons Attribution-NonCommercial-NoDerivatives 4.0 International (CC BY-NC-ND 4.0) License. 